\title{
“NORMAL” ORIENTATION DISTRIBUTIONS
}

\author{
Dedicated to Geoffrey S. Watson, Princeton, NJ, USA, \\ on the occasion of his 70th birthday
}

\section{H. SCHAEBEN}

\author{
Laboratory of Metallurgy of Polycrystalline Materials (LM2P), University of \\ Metz, Ile du Saulcy, 57045 Metz-Cedex 01, France
}

(Received January 10, 1992)

\begin{abstract}
Analogues of the normal distribution in Euclidean space for orientations represented by Rodrigues parameters are discussed. It is emphasized that different characterizations of the normal distribution in Euclidean space lead to different distributions in other spaces, none of which is mathematically superior to any other one. Particular analogues of the normal distribution are the Bingham distribution on $S_{+}^{4}$ for the purposes of mathematical statistics, and the Brownian motion distribution on $S_{+}^{4}$ in terms of probability theory and stochastic processes. It is reminded of the fact that a simple analogue of the central limit theorem in Euclidean space does not exist for the hyperspheres $S^{p}$ and projective hyperplanes $H^{p-1} \equiv S_{+}^{p}$.
\end{abstract}

KEY WORDS Characterizations of the normal distribution in Euclidean space; von Mises-Fisher matrix distribution on $S O(3)$; Bingham distribution, Brownian motion distribution on $H^{3} \equiv S_{+}^{4}$;

\section{INTRODUCTION}

Mathematical model distributions in the orientation space have recently been discussed by Matthies et al. (1988). In particular, the authors discuss the family of probability densities proportional to $\exp (\kappa \cos \tilde{\omega})$ labeled "standard Gaussian function" (Matthies, 1980), and in some length the family of the "truly normal distribution" given as infinite series expansion into characters of the irreducible representations of the group $S O(3)$ and $C$-coefficients. For the latter, the reader is referred to a communication in Russian by Savelova (1985) who is given credit for being the first to derive the $C$-coefficients of the "truly normal distribution".

The purpose of this short note is twofold. We would like to add some references which might be more easily accessible to the majority of the readership both in terms of language as well as mathematics. Furthermore, we would like to show how the two families relate to general normal theory and that they are in fact well known to mathematical statisticians and physicists for quite a while.

When discussing general normal, or synonymously, Gaussian theory it should be remembered that besides the characterization by the central limit theorem of probability theory there are many more characterization theorems for the normal distribution in Euclidean space (cf. Kagan et al., 1973; Mardia, 1975). These include characterizations in terms of moments, maximum likelihood, maximum entropy, infinite divisibility, invariance under orthogonal transformations etc. When James (1954) developed methods of normal multivariate analysis for the orthogonal group $O(p), p \in \mathbb{N}$, Stiefel and Grassmann manifolds he emphasized 
that much of the theory of normal multivariate analysis can be deduced from, or is closely related to the fact that the distribution of a normal multivariate sample is invariant under orthogonal transformations (James, 1954). With respect to stochastic processes or statistical physics the normal distribution in Euclidean space can be characterized in terms of random walk, Brownian motion, diffusion processes etc.

\section{THE VON MISES-FISHER-LANGEVIN DISTRIBUTION ON $S^{p}$}

What has been labeled "Gaussian standard function" (Matthies, 1980) can be written generally and without reference to the notion of orientations in texture analysis as

$$
f_{p}(\omega ; \kappa)=\left(c_{p}(\kappa)\right)^{-1} \exp (\kappa \cos \omega), \quad 0 \leq \omega \leq \pi
$$

Interpreting the angle $\omega$ as being defined by two unit vectors $\mu, x \in S^{p} \subset \mathbb{R}^{p}$ the density (1) can be rewritten explicitly as $p$-variate probability density

$$
f_{p}(x ; \kappa, \mu)=c_{p}(\kappa)^{-1} \exp \left(\kappa \mu^{t} x\right)
$$

of polar directional data. According to Watson (1983) it is referred to as von Mises (1918) for $p=2$, as Fisher (1953) for $p=3$, and as Langevin (1905) for any $p \in \mathbb{N}$, where it has arisen in his statistical-mechanical discussion of magnetism. The density (2) is rotationally symmetric with respect to its modal, or polar, vector $\mu \in S^{p}$; therefore, it is also called a central or zonal density.

Returning to the notion of rotations and orientations, and interpreting the angle $\omega, 0 \leq \omega \leq \pi$, as distance of orientations, the "Gaussian standard function" has been identified as the special case of the general von Mises-Fisher matrix distribution (Khatri and Mardia, 1977) on SO(3) when it is central (Schaeben, 1990), i.e. when it degenerates to the form (1). Maximum likelihood and information theoretic characterizations of the general Langevin distribution have been given by Mardia (1975). Thus, it is concluded that the Langevin distribution is the hyperspherical analogue of the normal distribution in Euclidean space for the purposes of mathematical statistics.

\section{THE BINGHAM DISTRIBUTION ON $S_{+}^{p} \equiv H^{p-1}$}

When applying the Rodrigues parameters of an orientation (cf. Altmann, 1986) it can be represented as a point on the upper fourdimensional hypersphere $S_{+}^{4}$. According to the one-one map of a matrix element of $S O(3)$ and a point on $S_{+}^{4}$ representing the same orientation, the corresponding one-one relation of the von Mises-Fisher matrix distribution on $S O(3)$ and the antipodally symmetric Bingham distribution (Bingham, 1964; 1974) on $S^{4}$ has explicitly been given (Prentice, 1986) and discussed in the context of texture analysis (Schaeben, 1990). If the von Mises-Fisher matrix distribution on $S O(3)$ degenerates to the Langevin distribution (1) of polar directions, then the Bingham distribution on $S_{+}^{4}$ degenerates to the rotationally symmetric Watson distribution (Bingham, 1964; Watson, 1965) of axial directions on $S_{+}^{4}$. Characterizations of the Bingham 
distribution in terms of maximum likelihood and information theory can be found in (Mardia, 1975). Thus, it is concluded that the Bingham distribution is the analogue of the normal distribution in Euclidean space on $S_{+}^{p}$, or more specifically on the $p$-1-dimensional projective hyperplane $H^{p-1}$, for the purposes of mathematical statistics. Prentice (1986) emphasized that the Bingham distribution provides distinct advantages as compared to the von Mises-Fisher matrix distribution for statistical applications thus confirming that the representation of orientations by Rodrigues parameters is suited best for statistical purposes (Moran, 1975; Altmann, 1986).

We should be able to represent any density on a hypersphere or projective hyperplane of some practical interest as a series expansion in terms of orthonormal functions on the hypersphere, i.e. in spherical harmonics and related functions (Watson, 1983).

The Langevin distribution (2) of polar directions on $S^{p} \subset \mathbb{R}^{p}, p \geq 3$, may be represented in terms of Fourier-Legendre series as

$$
f_{p}(\omega, \kappa)=\operatorname{area}^{-1}\left(S^{p}\right) \sum_{k=0}^{\infty} N_{k, p}\left[I_{k+(p-2) / 2}(\kappa) / I_{(p-2) / 2}(\kappa)\right] P_{k, p}(\cos \omega)
$$

with the modified Bessel functions $I_{v}(\kappa)$, the Legendre polynomials $P_{k, p}$ of order $k$ for $\mathbb{R}^{p}$, and the number

$$
N_{m, p}=(2 m+p-2) \frac{(m+p-3) !}{m !(p-2) !}
$$

of linearly independent homogeneous spherical harmonics of degree $m$ in $\mathbb{R}^{p}$, $p \geq 3$. The Legendre polynomials $P_{k, p}$ of order $k$ for $\mathbb{R}^{p}$ span the space of rotationally symmetric functions in $\mathscr{L}^{2}\left(S^{p}\right), p \geq 3$; for $p \geq 3$ they satisfy $c_{k}(p) P_{k, p}(t)=C_{k}^{((p-2) / 2)}(t)$ where $C_{k}^{(v)}(t)$ denotes the Gegenbauer function of order $k$ and with $c_{k}(p)=\Gamma(k+p-2) /(\Gamma(p-2) \Gamma(k+1))$ (Hartman \& Watson, 1974).

\section{THE BROWNIAN MOTION DISTRIBUTION ON $S^{p}$ AND $S_{+}^{p} \equiv H^{p-1}$}

The Brownian motion distribution (cf. Perrin, 1928; Arnold, 1941; Yosida, 1949; Roberts \& Ursell, 1960) on $S^{p} \subset \mathbb{R}^{p}, p \geq 3$, is given by its Fourier-Legendre series

$$
f_{p}(\psi, v)=\operatorname{area}^{-1}\left(S^{p}\right) \sum_{k=0}^{\infty} N_{k, p} \exp [-k(k+p-2) v] P_{k, p}(\cos \psi), \quad 0 \leq \psi \leq \pi(4)
$$

(Hartman \& Watson, 1974; Watson, 1983). In particular, this reads for $p=4$ when the hyperspherical angle $\psi$ is related to the angle of rotation $\omega$ of an 
orientation by $2 \psi=\omega$ (cf. Roberts \& Winch, 1984)

$$
\begin{aligned}
f_{4}(\psi, v) & =\left(2 \pi^{2}\right)^{-1} \sum_{k=0}^{\infty}(k+1)^{2} \exp [-k(k+2) v] P_{k, 4}\left(\cos \frac{\omega}{2}\right) \\
& =\left(2 \pi^{2}\right)^{-1} \sum_{k=0}^{\infty}(k+1) \exp [-k(k+2) v] C_{k}^{(1)}\left(\cos \frac{\omega}{2}\right) \\
& =\left(2 \pi^{2}\right)^{-1} \sum_{k=0}^{\infty}(k+1) \exp [-k(k+2) v] \frac{\sin \left[(k+1) \frac{\omega}{2}\right]}{\sin \frac{\omega}{2}}
\end{aligned}
$$

The last equation is an application of the identity $C_{k}^{(1)}(\cos \omega)=U_{k}(\cos \omega)$ where $U_{k}(\cos \omega)$ denotes the Chebyshev polynomial of the second kind.

Following an argument introduced by Arnold (1941) the Brownian motion distribution on the projective hyperplan $H^{p-1} \equiv S_{+}^{p} \subset \mathbb{R}^{p}$ is given by

$$
\begin{aligned}
h_{p}(\psi, v) & =f_{p}(\psi, v)+f_{p}(\pi-\psi, v) \\
& =\operatorname{area}^{-1}\left(S^{p}\right) \sum_{k=0}^{\infty} N_{2 k, p} \exp [-2 k(2 k+p-2) v] P_{2 k, p}(\cos \psi)
\end{aligned}
$$

which reads for $p=4$ and $0 \leq \psi \leq \pi / 2$

$$
\begin{aligned}
h_{4}(\psi, v) & =\left(4 \pi^{2}\right)^{-1} \sum_{k=0}^{\infty}(2 k+1)^{2} \exp [-2 k(2 k+2) v] P_{2 k, 4}\left(\cos \frac{\omega}{2}\right) \\
& =\left(4 \pi^{2}\right)^{-1} \sum_{k=0}^{\infty}(2 k+1) \exp [-k(k+1) 4 v] \frac{\sin \left[(2 k+1) \frac{\omega}{2}\right]}{\sin \frac{\omega}{2}}
\end{aligned}
$$

Applying a characterization of the normal distribution in terms of infinitely divisible distributions, Savelova (1984) [in English] derived for $S O(3)$ the central orientation distribution depending on the orientation distance $\tilde{\omega}$

$$
f(\tilde{\omega}, v)=\sum_{k=0}(2 k+1) \exp \left[-k(k+1) v^{2}\right] D_{k}(\tilde{\omega})
$$

in terms of generalized Legendre series expansion, or more specifically in terms of series expansion into characters of the irreducible representations of the group $S O(3)$ with the Dirichlet kernel

$$
D_{n}(\tilde{\omega})=1+2 \sum_{k=1}^{n} \cos (k \tilde{\omega})=\left\{\begin{array}{ll}
\frac{\sin ((2 n+1) \tilde{\omega} / 2)}{\sin (\tilde{\omega} / 2)} & \tilde{\omega} \neq 2 j \pi \\
2 n+1 & \tilde{\omega}=2 j \pi
\end{array} \quad j \in \mathbb{Z}\right.
$$

which is obviously identical with the Brownian motion distribution $h_{4}$ on $S_{+}^{4}$.

Thus, the Brownian motion distribution $h_{4}$ on $S_{+}^{4}$ or equivalently the distribution (6) derived by Savelova (1984) is the analogue of the normal distribution in Euclidian space in terms of probabilistic arguments, i.e. in probability theory (cf. Watson, 1983). 
Matthies et al. (1988) apply arguments of the central limit theorem in Euclidean space to derive the distribution (6) in an oversimplified way. Then they relate it to the central limit theorem for $S O(3)$ which they seem to imagine as a simple analogue of the central limit theorem in Euclidean space. However, such an analogue does in general not exist for arbitrary manifolds; a simple analogue of the central limit theorem in Euclidean space for hyperspheres $S^{p} \subset \mathbb{R}^{p}$, projective hyperplanes $H^{p-1} \subset \mathbb{R}^{p}$, or for the group $S O(p), p \in \mathbb{N}$, would result in the corresponding uniform distributions (Breitenberger, 1963; Roberts \& Winch, 1984; Watson, 1991).

\section{CONCLUSIONS}

It should be obvious that there is no unique analogue for arbitrary spaces and manifolds of the normal distribution in Euclidean space (Watson 1983) nor that any analogue should generally be preferred as truly normal to any other one. Interpretations of orientation densities in terms of mechanisms of texture development related to an imaginary simple analogue of the central limit theorem for $S O(3)$, or $S_{+}^{4} \equiv H^{3}$ respectively, may be misleading.

\section{ACKNOWLEDGMENT}

This research was funded by a grant for proposition no. ERB4040PL900089 from the EC plan SCIENCE under contract no. ERBSC1*CT005001. Discussions of the subject of this short note with Geoffrey S. Watson, Princeton, are greatfully appreciated. Special thanks are due to G. Posanski, University of Technology at Aachen, FRG, who brought the translation (Savelova, 1984) to my attention.

\section{References}

1. Altmann, S. L., (1986). Rotations, Quaternions, and Double Groups; Oxford University Press, Oxford.

2. Arnold, K. J. (1941). Ph.D. Thesis, Massachusetts Institute of Technology, USA.

3. Bingham, C. (1964). Ph.D. Thesis, Yale University, USA.

4. Bingham, C. (1974). Ann. Statist., 2, 1201-1225.

5. Breitenberger, E. (1963). Biometrika, 50, 81-88.

6. Fisher, R. A. (1953). Proc. Roy. Soc. ser. A, 217, 295-305.

7. Hartman, P. and Watson, G. S. (1974). Ann. Prob., 2, 593-607.

8. James, A. T. (1954). Ann. Math. Stat., 25, 40-75.

9. Kagan, A. M., Linnik, Yu K. \& Rao, C. R. (1973). Characterization problems in mathematical statistics: J. Wiley \& Sons, New York.

10. Khatri, C. G. \& Mardia, K. V., 1977, J. Roy. Statist. Soc., ser B, 39, 95-106.

11. Langevin, P. (1905). Ann. de Chim. et de Phys., 5, 70-127.

12. Mardia, K. V. (1975). In Patil, G. P., Kotz, S., \& Ord, J. K., (eds.), Statistical Distributions in Scientific Work, vol. 3, D. Reidel Publishing Company, Dordrecht, 365-385.

13. Matthies, S. (1980). Phys. Stat. Sol. (b), 101, K111-K115.

14. Matthies, S., Muller, J., \& Vinel, G. W., 1988, Textures and Microstructures, 10, 77-96.

15. Mises, R. von (1918), Physikalische Zeitschrift, 19, 490-500.

16. Moran, P. A. P. (1975). In Gani, J., (ed.), Perspectives in Probability and Statistics, Applied Probability Trust, 295-301. 
17. Perrin, F. (1928). Annales Scientifique de l'ecole normale superieure, 45, 1-51.

18. Prentice, M. J. (1986). J. Roy. Statist. Soc., ser. B, 48, 214-222.

19. Roberts, P. H. \& Ursell, H. D. (1960). Phil. Trans. Roy. Soc. London, ser A, 252, 317-356.

20. Roberts, P. H. and Winch, D. E. (1984). Adv. Appl. Prob., 16, 638-655.

21. Savelova, T. I. (1984). Industrial Laboratory, 50, 468-474; translated from Zavodskaya Laboratoriya, 50, 48-52.

22. Savelova, T. I., 1985, Preface to Novye Metody Issledovanija Tekstury Polikristallicheskich Materialov: Metallurgija, Moscow [in Russian].

23. Schaeben, H. (1990). Textures and Microstructures, 13, 51-54.

24. Watson, G. S. (1965). Biometrika, 52, 193-201.

25. Watson, G. S. (1983). Statistics on Spheres: J. Wiley \& Sons, New York.

26. Watson, G. S. (1991). Pers. comm.

27. Yosida, K. (1949). Ann. Math. Stat., 20, 292-296. 